\title{
SOBERANÍA ALIMENTARIA EN ECUADOR: FUNDAMENTOS TEÓRICOS Y METODOLÓGICOS PARA UN MODELO DE MEDICIÓN
}

\section{FOOD SOVEREIGNTY IN ECUADOR: THEORETICAL AND METHODOLOGICAL BASES FOR A MEASUREMENT MODEL}

Arnaldo Andrés Vergara Romero, Mgtr. Magíster en Economía mención en Desarrollo Económico y Políticas Públicas (Ecuador). arvergara@mgs.ecotec.edu.ec

Adrián Nixon Moreno Silva, Mgtr. Magíster en Economía y Dirección de Empresas (Ecuador). Docente Tiempo Completo de la Facultad de Ciencias Económicas y Empresariales de la Universidad Tecnológica ECOTEC, Ecuador. amoreno@ecotec.edu.ec

\section{ARTÍCULO DE INVESTIGACIÓN}

Recibido: 21 de agosto de 2019.

Aceptado: 15 de noviembre de 2019.

\section{RESUMEN}

El presente artículo contiene una revisión teórica para fundamentar una metodología preliminar de los indicadores que, permitan medir el concepto de Soberanía Alimentaria en Ecuador mediante un modelo estadístico, buscando las variables óptimas para dicha medición y a su vez variables indicadoras que regulen dicho modelo y los resultados esperados. La metodología que se propone en la investigación es cuantitativa, correlacional y causal, con recolección de datos de fuentes secundarias mediante bases especializadas en materia de Soberanía Alimentaria que sustentan al Banco Mundial. Mediante técnicas de análisis multivariado se comprobará la fiabilidad de los datos y la comprobación del modelo por la técnica de Múltiples Indicadores y Múltiples Causas (MIMIC) con el paquete informático RStudio.

Palabras clave: soberanía alimentaria, modelo MIMIC, desnutrición, políticas alimentarias. 


\section{ABSTRACT}

This article contains a theoretical review to support a preliminary methodology of the indicators that allow measuring the concept of Food Sovereignty in Ecuador through a statistical model, looking for the optimal variables for said measurement and in turn indicator variables that regulate said model and expected results. The methodology proposed in the research is quantitative, correlational and causal, with data collection from secondary sources through specialized bases on Food Sovereignty that support the World Bank. By means of multivariate analysis techniques, the reliability of the data and the verification of the model will be checked by the Multiple Indicators and Multiple Causes (MIMIC) technique with the RStudio software package.

Keywords: food sovereignty, MIMIC model, undernourishment, food policies.

\section{INTRODUCCIÓN}

La importancia de los alimentos para la vida cotidiana puede ser exagerada, ya que todo el mundo tiene que alimentarse para poder vivir. Por lo tanto, la relación critica entre sociedad, naturaleza y los problemas dentro y entre sociedades con el sistema agroalimentario se vuelven particularmente visibles (Anderson \& Bellows, 2012). Sin embargo, el debate público generalmente se limitó con analizar el problema alimentario desde una forma individual y aislada, en la actualidad se nota que el sistema agrícola y alimentario está estrechamente vinculado a las principales crisis modernas que conlleva la pobreza, el hambre, la salud, el clima, la energía, la pérdida de biodiversidad y recursos. Entre los principales recursos fundamentales de la vida que se encuentran en el área de tensión entre el uso excesivo, la degradación y la concentración de estos recursos por países desarrollados se tiene a la tierra, el conocimiento ancestral, las semillas y el agua (Godfray et al., 2010).

El debate sobre la crisis alimentaria mundial de la última década ha alcanzado nuevas dimensiones (Simon Reardon \& Pérez, 2010). La cantidad de personas con hambre en todo el mundo sigue aumentando, a pesar de oponerse a objetivos políticos globales. Las razones de esto son cuestiones como la especulación alimentaria, el acaparamiento mundial de la tierra, la destrucción del Medio Ambiente y el cambio climático. No obstante, en este debate no se pierde de viste las causas centrales de los problemas a largo plazo como la creciente orientación del mercado mundial a los modelos neoliberales, explotación masiva de recursos y la valorización capitalista de la agricultura (Loke \& Leung, 2013). 
Bajo este abordaje de los debates sobre alimentación nace la ONG La Vía Campesina y en el año 1996 en el Foro de la Cumbre Mundial sobre la alimentación presenta la Soberanía Alimentaria, en ese momento hasta la actualidad adoptada y defendida cada vez más por organizaciones campesinas, organizaciones de la sociedad civil y pueblos indígenas, convirtiéndola en un movimiento social transnacional notable y creciente (Rosset, 2016).

Existen siete naciones que han incluido el Concepto de Soberanía Alimentaria en sus reformas constitucionales y/o leyes nacionales, se incluyen Venezuela en 1999/2008, Senegal en 2004, Malí en 2006, Nepal 2007, Ecuador en 2008, Bolivia y Nicaragua en 2009. Como propuestas de reformas legislativas para la inclusión de la Soberanía Alimentaria en las políticas alimentarias están México, El Salvador, República Dominicana y Perú. Además, se han aprobado políticas locales de varios condados de Estados Unidos e iniciativas en Canadá.

(Godek, 2014, p. 3)

La visión de la Soberanía Alimentaria es que llegar a un mundo sin hambre y pobreza, solo es posible si los productores de alimentos y las personas hambrientas están incluidas en las decisiones claves y no debe estar sujeto solo a la lógica del mercado. La Soberanía Alimentaria fue propuesta por La Vía Campesina en 1996 y a su vez fue incorporada en el debate mundial por la Organización de las Naciones Unidas para la alimentación y la agricultura (FAO) en la $32^{\mathrm{a}}$ Conferencia Regional de la FAO para América Latina y el Caribe y a su vez una Ley Marco de derecho a la alimentación, seguridad y soberanía alimentaria (FAO, 2013, p. 3), aprobada en la XVIII Asamblea Ordinaria del Parlamento Latinoamericano y Caribeño desde el 30 de noviembre al 1 de diciembre de 2012, en la ciudad de Panamá (Parlamento Latinoamericano, 2012).

La finalidad del trabajo es identificar los conceptos e indicadores relevantes de Soberanía Alimentaria propuestos por organismos internacionales, nacionales y de estudios previos para que se pueda aproximar una medición para el Ecuador.

\section{Modelos para políticas alimentarias en base al derecho a la alimentación}

La alimentación es un tema complejo, distribuido en muchas disciplinas académicas, el estudio de los alimentos evoluciona continuamente con nuevas direcciones, metodologías, modelos e hipótesis. Por lo tanto, la confusión sobre conceptos de alimentos compite entre sí (Loke \& Leung, 2013). 
El derecho a la alimentación, codificado en el Artículo 25 de la Declaración Universal de los Derechos Humanos de 1948 y en otros tratados internacionales, constituye la base ética y normativa internacional de la política alimentaria mundial. Dentro del complejo régimen y derecho de la alimentación en el mundo se han establecido varios enfoques normativos que, algunos de los cuales se complementan entre sí sus conceptos.

El primer enfoque es la Seguridad Alimentaria y Nutricional que se basa en el aumento de la producción, la expansión de las tierras agrícolas y la intensificación de la agricultura. Estas políticas ya fueron utilizadas en el contexto de la "revolución verde" ${ }^{1}$ y han llevado a un aumento masivo de la producción agrícola en los países de desarrollo, pero al mismo tiempo causaron graves daños ambientales que se han ignorado en el tiempo. Para López \& Franco (2015), este enfoque crea un modelo que "predomina el aumento de la producción, la gestión del riesgo agrícola, la gerencia de recursos, el libre comercio, los medios de subsistencia a nivel mundial, el acceso, la distribución y la sostenibilidad de los sistemas alimentarios" (p. 1362).

El segundo enfoque es la Soberanía Alimentaria que se basa en la premisa de que la Seguridad Alimentaria y Nutricional requiere una reestructuración de las políticas alimentarias mundiales, siendo posible si se eliminan las desigualdades políticas y económicas básicas fortaleciendo las estructuras agrícolas a pequeña escala y los mercados regionales, así como los métodos de producción ecológicos. Según López y Franco, "se enfatiza en la producción local, la protección de la biodiversidad, la equidad, la sostenibilidad ambiental, los modelos agroecológicos y la participación de la sociedad civil, entre otros" (p. 1362).

La Seguridad Alimentaria y la Soberanía Alimentaria son conceptos claves en la discusión sobre cómo lograr alimentar al mundo de manera suficiente, sostenible y saludable. Estos conceptos se centran en diferentes actores y dimensiones para la producción de los alimentos. Bajo el contexto Latinoamericano se busca crear una seguridad alimentaria que conduzca a la Soberanía Alimentaria y no una Seguridad Alimentaria sin ser soberano. Si se tiene Soberanía Alimentaria se puede decidir cómo producir, qué variantes utilizar y cómo alimentarse.

\footnotetext{
${ }^{1}$ Término acuñado al incremento productivo mediante las tecnologías mencionadas por Norman E. Borlaug y estudiado por González (2006)
}

ISSN: 1390-9320, Edición Especial, diciembre 2019 
La Soberanía Alimentaria puede acordar un enfoque conveniente para disminuir la incidencia de la inseguridad crónica y los problemas socioambientales que aquejan al mundo, aplicables a niveles global, regional, nacional y local.

\section{Conceptualización de la soberanía alimentaria}

La Soberanía alimentaria es un concepto dinámico en constante evolución. Desde que se propuso por primera vez, ha evolucionado con la transformación del contexto internacional y su adopción por parte de una red global de movimientos sociales y organizaciones de la sociedad civil para incluir los intereses de una amplia gama de colectivos $\mathrm{y}$, al mismo tiempo adaptarse a diferentes contextos locales (Binimelis et al., 2014).

Para Simon Reardon \& Pérez (2010) la Soberanía Alimentaria "está guiada por el derecho de las personas y sus gobiernos a definir democráticamente la composición de sus sistemas de producción agraria y agrícola sin interferencia externa."

El mundo agrario siempre ha tenido la resistencia de abajo hacia arriba, desde los agricultores hasta los que planifican políticas, por esto la Soberanía Alimentaria se considera una definición política que:

se refiere al derecho de un país a definir de manera autónoma sus políticas agrícolas y alimentarias, así como sus patrones de producción y consumo, de una manera socialmente justa y respetuosa con el medio ambiente, lo que permite a los ciudadanos y habitantes de un área acceder a alimentos sostenibles y promover el desarrollo de la zona en la que viven. (Butti Al Shamsi, Compagnoni, Timpanaro, Cosentino, \& Guarnaccia, 2018)

Botella Rodríguez (2018) analiza la importancia de la Soberanía Alimentaria y menciona que:

Se basa en revitalizar la agricultura familiar y campesina, así como su contribución a la producción nacional de alimentos con tecnologías poco dependientes de insumos externos, maquinaria y tecnología importada, la sustitución de alimentos importados y el acceso mejorado a la tierra y los mercados domésticos (p. 6).

Los elementos que delimitan a la Soberanía Alimentaria son: (i) una lucha global a la agricultura industrial y sus consecuencias ecológicas; (ii) la defensa de un "modo campesino" como base de un sistema alimentario sostenible y socialmente justo; y (iii) un programa para concretar el objetivo mundial-histórico (Bernstein, 2015). 
Se puede resumir que la Soberanía Alimentaria se centra en la cuestión de cómo se producen los alimentos, bajo qué control se lleva a cabo, en qué condiciones sociales y ambientales se desarrollan, y por lo tanto exige la politización de las relaciones de producción.

\section{Dimensiones de la soberanía alimentaria}

La Soberanía Alimentaria es un concepto que ha ganado cada vez más la atención de académicos, investigadores y profesionales del desarrollo que enlazan muchas cuestiones dentro de la política alimentaria de hoy (Altieri, 2011). Las prioridades de la cooperación internacional deben ser redefinidas, especialmente en el área de políticas globales y el aspecto de desarrollo sostenible ecológica y socialmente, haciendo viable un retorno a nivel de desarrollo local.

Dando la importancia al contexto global y cultural de la producción de alimentos, se analiza las dimensiones bajo el marco analítico de Calix de Dios et al. (2014) que se resume en la figura 1. En esta figura se observan las dimensiones en una noción relativa y cíclica, asociada a una compleja variedad de factores (socioeconómicos, geopolíticos, endógenos y exógenos) y nuevas realidades (política de tierras, cuestiones de políticas externas, etc.) relacionadas, en particular con los mercados agrícolas en el contexto internacional cada vez más incierto. Todos estos cambios que se evidencian hoy requieren un nuevo grado de flexibilidad que incluye a la Soberanía Alimentaria de forma de investigación y puesta en práctica en nuestra sociedad. 
Figura 1. Marco analítico de las dimensiones de la Soberanía Alimentaria. Fuente: Calix de Dios et al. (2014).

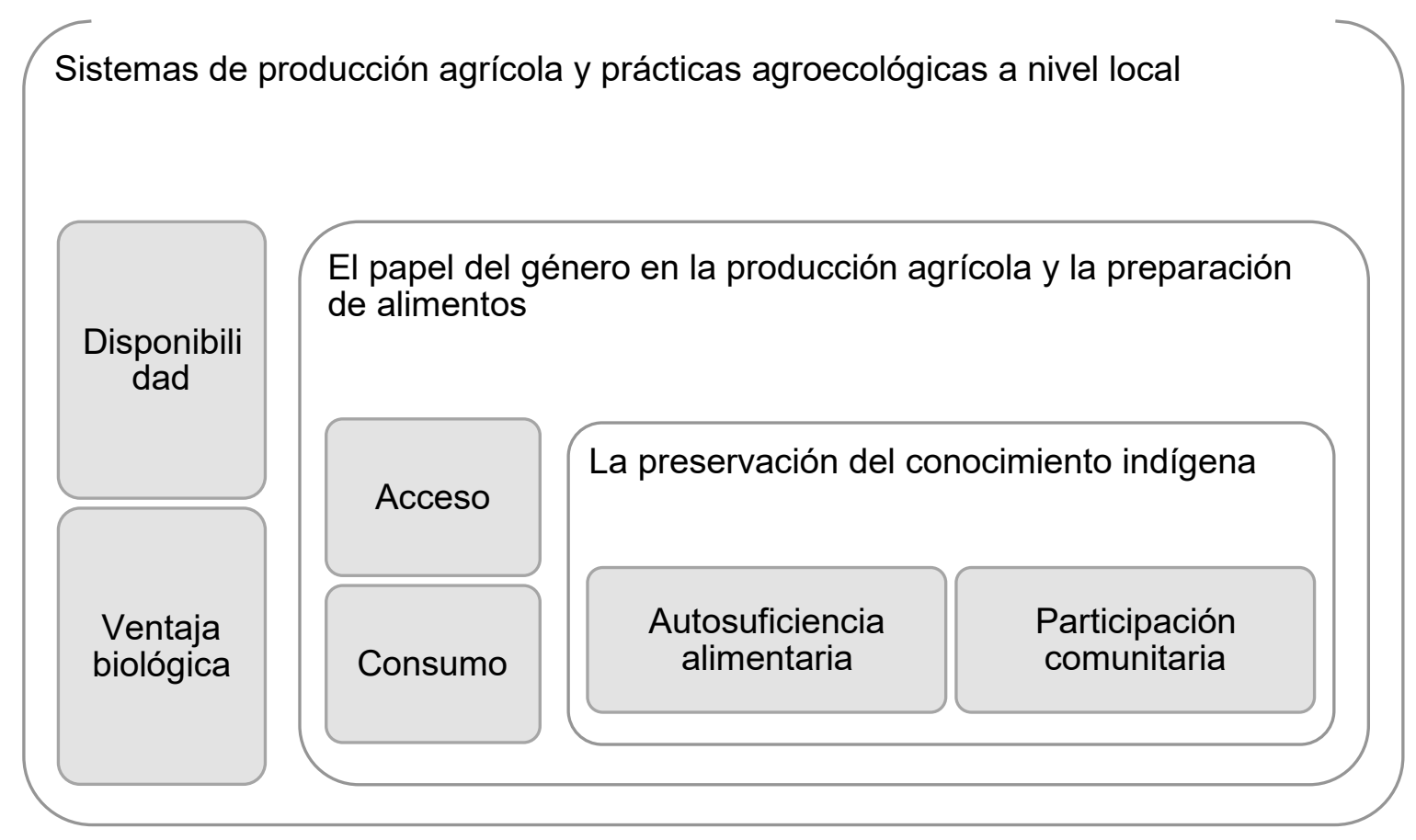

Fuente: Elaboración propia.

\section{Soberanía alimentaria en Ecuador}

En el Ecuador desde la década de 1990 se observa la afectación de millones de personas de zonas rurales que abandonaron la agricultura y emigraron a centros urbanos y otros países en busca de mejorar su calidad de vida, girando las políticas públicas en torno a la pobreza y el hambre. Sin embargo, la conciencia de las consecuencias perjudiciales y no deseadas de la modernización agrícola impulso la protesta social sobre los regímenes alimentarios hegemónico (Villegas, 1998).

El gobierno ecuatoriano con el afán de modernizar las ideas y modelos no convencionales en tema agropecuarios se convierte en el primer País en incorporar la Soberanía Alimentaria es su Carta Magna, convirtiéndose en un caso de estudio muy interesante para entender el Marco teórico de este concepto y su relación con el país y sus movimientos sociales (Akchurin, 2015).

La Constitución del Ecuador (2008), en su artículo 281 menciona que "la Soberanía Alimentaria constituye un objetivo estratégico y una obligación del Estado para garantizar que 
las personas, comunidades, pueblos y nacionalidades alcancen la autosuficiencia de alimentos sanos y culturalmente apropiado de forma permanente." (página 138)

En el contexto de esta garantía constitucionales podemos observar factores críticos para transformar las políticas agrícolas y alimentarias de la época en favor de una producción agrícola sostenible guiada por justicia social y ambiental.

Bajo esta premisa el Gobierno Ecuatoriano amplía sus conceptos y estrategias mediante la Ley Orgánica del Régimen de la Soberanía Alimentaria (LORSA, 2009) indica los principios de aplicación de la ley en su artículo 4:

Esta ley se regirá por los principios de solidaridad, autodeterminación, transparencia, no discriminación, sustentabilidad, sostenibilidad, participación, prioridad del abastecimiento nacional, equidad de género en el acceso a los factores de la producción, equidad e inclusión económica y social, interculturalidad, eficiencia e inocuidad, con especial atención a los microempresarios, microempresa o micro, pequeña y mediana producción (p. 2).

En el cuerpo jurídico analizado en el párrafo anterior, podemos observar que se distingue en categorizaciones bien definidas a las que podemos asociarlas a dimensiones y componentes, haciendo hincapié que estas son las metas de la Soberanía Alimentaria propuestas para el Ecuador, tal como se observa en la figura 2.

Figura 2. Categorización de dimensiones en la LORSA. Fuente: (LORSA, 2009).

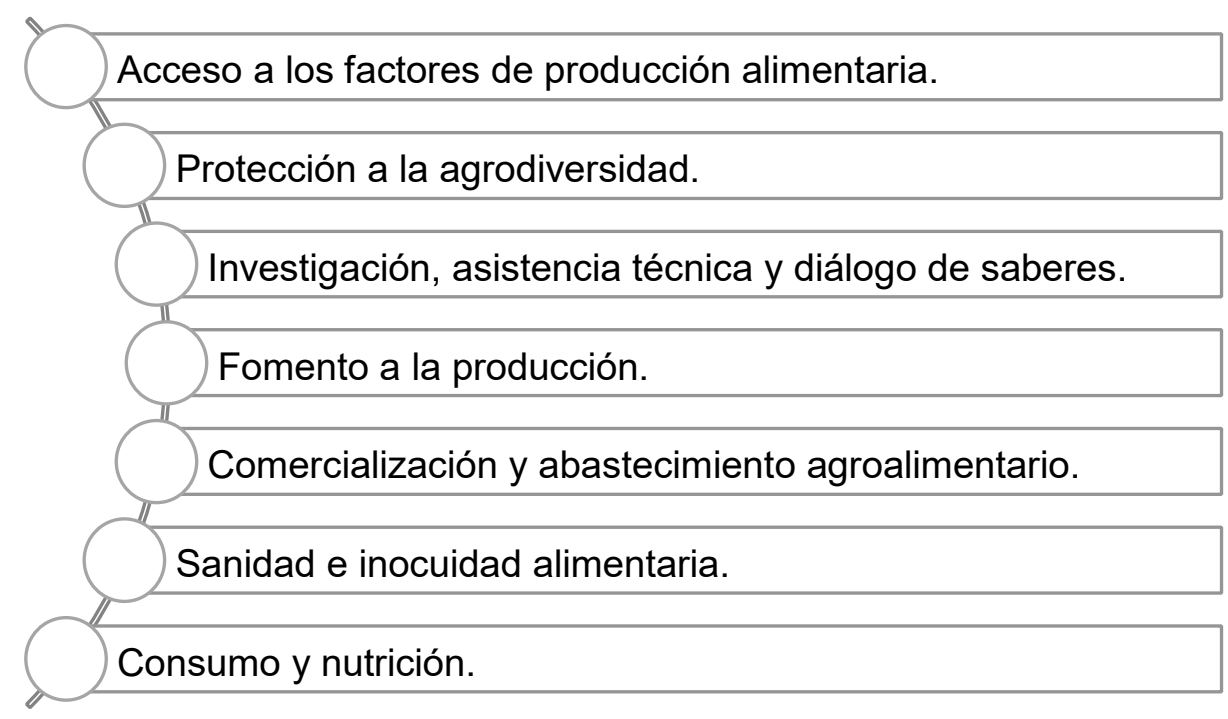

Fuente: Elaboración propia. 
Las dimensiones y componentes en este cuerpo jurídico tienen muchas semejanzas con las dimensiones analizadas en el concepto de Soberanía Alimentaria. Lo que pone rumbo a la investigación para analizar variables cuantificadas por organismos internacionales y nacionales de estadísticas y su comportamiento en su entorno natural.

Para el efectivo monitoreo en el Ecuador se rige por organismos internacionales como la Organización de Naciones Unidas establecieron en la Agenda 2030 los Objetivos de Desarrollo Sostenible, involucrando el segundo objetivo poner fin al hambre, lograr la seguridad alimentaria y la mejora de la nutrición y promover la agricultura sostenible. Esto se da porque el sector alimentario y el sector agrícola ofrecen soluciones claves para el desarrollo y son vitales para la eliminación del hambre y la pobreza (ONU, 2019).

Sin embargo, a nivel local la Soberanía Alimentaria también está inmersa en el Plan Nacional de Desarrollo 2017-2021 en su eje 2 de Economía al Servicio de la Sociedad, considerado en el objetivo 6 que lograría desarrollar las capacidades productivas y del entorno para lograr la Soberanía Alimentaria y el Buen Vivir rural. Estas metas se deben alcanzar revisando políticas y monitoreando indicadores claves de alimentación (SENPLADES, 2017).

Entre los organismos que monitorean las políticas de Soberanía Alimentaria se tiene al Ministerio de Agricultura, Ganadería, Acuacultura y Pesca, "es la institución rectora del sector agropecuario, encargada de la articulación de los servicios financieros y no financieros, facilitando el desarrollo de los mercados de servicios no financieros, a través de la política pública para la agricultura familiar campesina priorizando los servicios de comercialización, asociatividad e innovación, para mejorar las condiciones de vida de la población, garantizando la soberanía alimentaria" (MAGAP, 2019).

EI MAGAP no solo enmarca la Soberanía Alimentaria en su visión y misión, tiene incorporado una estructura de objetivos y ejes estratégicos en torno a esta garantía constitucional.

La Conferencia Plurinacional e Intercultural de Soberanía Alimentaria tiene de misión incidir “la construcción, implementación y acompañamiento participativo al cumplimiento de las políticas públicas y del Sistema de Soberanía Alimentaria y Nutricional (SISAN), a través de un amplio proceso de diálogo, coordinación y articulación, entre la Sociedad Civil y Estado, para la implementación de la Soberanía Alimentaria" (COPISA, 2019). 
Dentro de la organización de COPISA, se conforma por 9 representantes según la figura 3 designado por el Consejo de Participación Ciudadana y Control Social (CPCCS), para poder ejercer las atribuciones que por Ley les toca regular y proponer.

COPISA es la organización que formula y concientiza a todos los sectores que tienen representación en ella, orientando al conocimiento y propuestas de sectores casi vulnerados por el sistema agroalimentario dominante. Esta entidad tiene un presupuesto por parte del Estado y es la que lleva las opiniones y/o reclamos de la Sociedad civil hacia el poder ejecutivo y legislativo.

Figura 3. Conformación de los Representantes de COPISA.

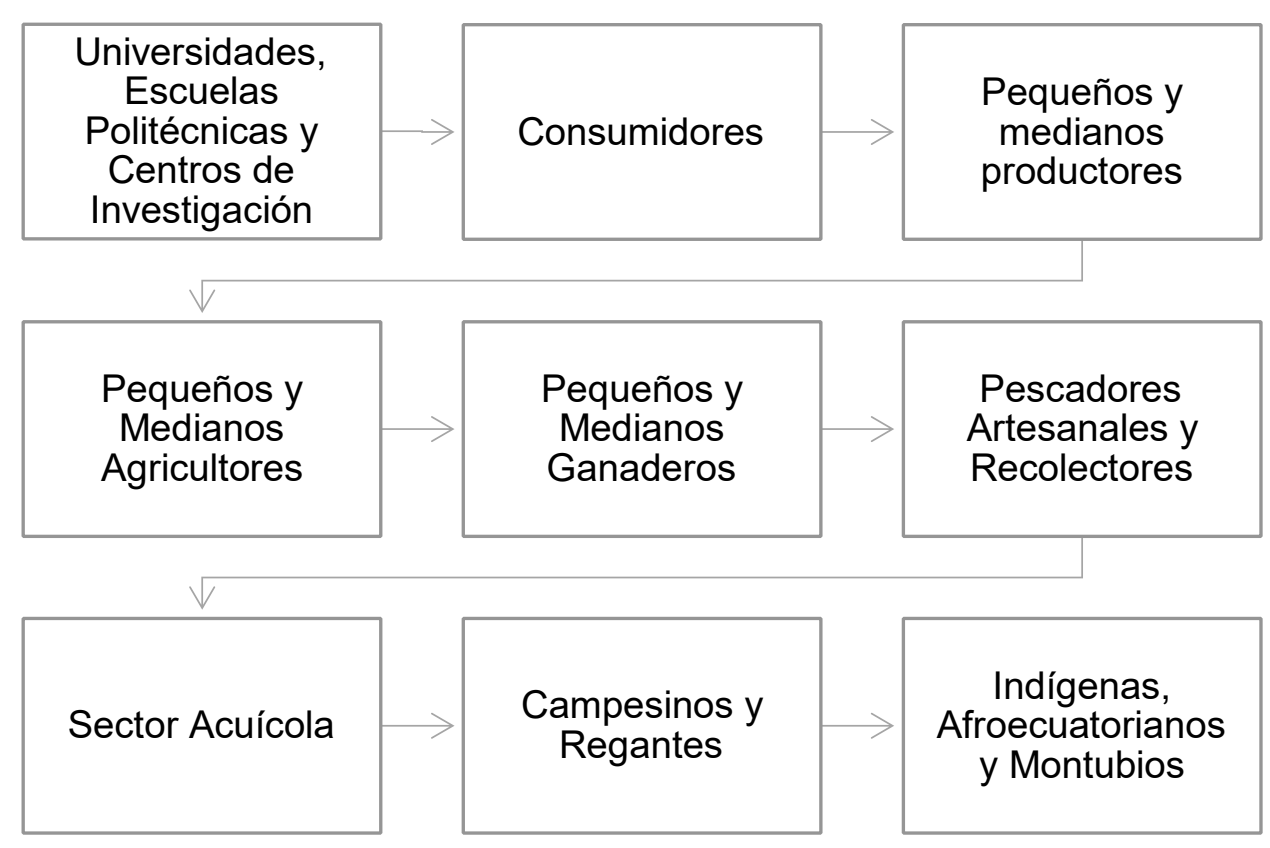

Fuente: (COPISA, 2019). Elaboración propia.

La Confederación Nacional de Organizaciones Campesinas, Indígenas y Negras (FENOCIN), es la agrupación de 60 organizaciones de segundo grado, 1300 organizaciones sociales, 2200 comunidades de base y aproximadamente 500 mil familias inscritas. La misión de la FENOCIN (2019) es:

Es la promoción integral y colectiva de hombres y mujeres del sector rural y urbano marginal, participa y aporta activamente de la construcción de una sociedad, equitativa 
y solidaria que mejore la calidad de vida que eleve su autoestima, su creatividad, sus conocimientos y destrezas, su capacidad crítica y reflexiva, conservando su propia identidad.

Con una transparencia e integral de desarrollo, con un alto grado de resolución de conflictos, nivel elevado de inserción y concertación con otros sectores sociales y gubernamentales, con una participación equitativa de mujeres y jóvenes en la dirección política, económica, social e intercultural.

Esta organización que comprende a casi todos los movimientos sociales del Ecuador, es el gran impulsador de los cambios agrarios que están contemplados en la Constitución, en la actualidad es el veedor más importante de la Soberanía Alimentaria en el territorio ecuatoriano.

En el Ecuador las políticas públicas a nivel de menor escala como son los Gobiernos Autónomos Descentralizados (GAD) Parroquiales están siendo impulsadas por COPISA y alineados a los Objetivos de Desarrollo y el Plan Nacional de Desarrollo 2017-2017. En la tabla 1 se puede observar las principales políticas que fueron firmadas mediante convenio, siendo desarrolladas y ejecutadas en el territorio ecuatoriano.

Tabla 1. Políticas Públicas 2012-2018

\begin{tabular}{|c|c|c|c|c|c|}
\hline AÑO & PARROQUIA & CANTÓN & AÑO & PARROQUIA & CANTÓN \\
\hline \multirow{12}{*}{2013} & Isla Bejucal & Baba & \multirow{12}{*}{2015} & Las Lojas & Daule \\
\hline & Macuma & Morona & & Juan Bautista Aguirre & Daule \\
\hline & Antonio Sotomayor & Vinces & & Limonal & Daule \\
\hline & García Moreno & Pelileo & & El Laurel & Daule \\
\hline & Angochagua & Ibarra & & Cone & Yaguachi \\
\hline & Toacazo & Latacunga & & Virgen de Fátima & Yaguachi \\
\hline & Santiago & Loja & & Sabanilla & Pedro Carbo \\
\hline & Guare & Baba & & La Guayas & El Empalme \\
\hline & San Lucas & Loja & & San Carlos & Quevedo \\
\hline & San Andrés & Píllaro & & Patricia Pilar & Buena Fe \\
\hline & La Esperanza & Pedro Moncayo & & San José de Ancón & Santa Elena \\
\hline & Simón Bolívar & Pastaza & & Anconcito & Santa Elena \\
\hline
\end{tabular}




\begin{tabular}{|c|c|c|c|c|c|}
\hline 2016 & Guasuntos & Alausí & 2018 & Nuevo Paraíso & Francisco de Orellana \\
\hline 2017 & El Dorado & Francisco de Orellana & & San Andrés de Taday & Azogues \\
\hline
\end{tabular}

Fuente: Elaboración propia a partir de COPISA (2019).

Las políticas públicas que se están promoviendo es la protección a la población del consumo de alimentos contaminados que pongan en riesgo la salud humana y animal, parroquias libres de agroquímicos tóxicos, parroquias libres de desnutrición infantil, parroquias sin suelos degradados ni erosionados, parroquias agroecológicas y con biodiversidad nativa en recuperación.

COPISA también firmó una Política pública de Soberanía Alimentaria reformas de productos financieros de BanEcuador, que garanticen el acceso a créditos diferenciados para el sector pesquero artesanal, esta entidad ha elaborado propuestas de leyes en temas de agrodiversidad, agroindustria, comunas, créditos, consumo, comercialización, pesca, tierras y sanidad desde el 2012 hasta la actualidad.

\section{MATERIALES Y MÉTODOS}

El tipo de investigación que se utilizará es de enfoque cuantitativo con paradigma hipotéticodeductivo, cuyo diseño será correlacional-explicativo-predictivo, porque inicia el análisis de las variables mediante la fundamentación teórica y revisión de estudios previos para definir las variables que se ajusten a la definición de Soberanía Alimentaria y luego se realizará un análisis estadístico robusto para ver la relación causa-efecto de las variables observables, latentes y de control.

Se utilizará como técnica de recolección a la revisión documental y de la base de datos del Banco Mundial (BM) que será consultada de la página web oficial, y es alimentada por varias instituciones públicas y/o privadas con sus respectivas especializaciones, entre las principales se pueden observar en la figura 4. 
Figura 4. Instituciones que sustentan la Base de Datos del Banco Mundial.

\begin{tabular}{|c|}
\hline $\begin{array}{l}\text { División de Estadísticas de las Naciones Unidas. (Base de datos } \\
\text { Comtrade) }\end{array}$ \\
\hline Centro de Análisis de Información sobre Dióxido de Carbono. \\
\hline $\begin{array}{l}\text { División de Ciencias Ambientales del Laboratorio Nacional de Oak Ridge. } \\
\text { (Tennesse, USA) }\end{array}$ \\
\hline División de Población de las Naciones Unidas. \\
\hline $\begin{array}{l}\text { Secretaría de la Comunidad del Pacífico: Programa de Estadísticas y } \\
\text { Demografía. }\end{array}$ \\
\hline $\begin{array}{l}\text { Oficinas nacionales de estadísticas mediante los informes de censos. } \\
\text { (OCDE) }\end{array}$ \\
\hline $\begin{array}{l}\text { Organización de las Naciones Unidas para el Desarrollo Industrial. } \\
\text { Organización de las Naciones Unidas para la Agricultura y la } \\
\text { Alimentación. (FAO) }\end{array}$ \\
\hline
\end{tabular}

Fuente: Elaboración propia.

Como muestra se explorará todos los indicadores de las bases de datos BM desde el periodo 1985 hasta el 2017. La información será procesada en el Programa estadístico RStudio, con los paquetes lavaan y semplot.

Se estimará verificar una variable latente para la comprensión del fenómeno denominado Soberanía Alimentaria, para dicha estimación o modelización empírica se utilizará el modelo estadístico matemático de análisis multivariado, denominado Múltiples Indicadores y Múltiples Causas. (MIMIC)

El enfoque MIMIC es una variante del Modelo de Ecuaciones Estructurales (SEM, por sus siglas en inglés), este enfoque es el más completo, ya que se basa en un modelo de comportamiento bien estructurado (Kline, 2011). El enfoque MIMIC se ajusta a los objetivos de este trabajo de investigación, porque puede incorporar diferentes alternativas o indicadores complementarios de la Soberanía Alimentaria es un proceso de estimación, mediante el modelo, no solo se pueden obtener estimación de parámetros para el caso ecuatoriano, sino que también se pueden estimar los efectos de ciertos cambios en las políticas alimentarias durante el periodo de elaboración y de transición (Trebicka, 2014). 
El modelo MIMIC consta de dos partes, la ecuación estructural (1) y el sistema de ecuaciones de medición (2). El modelo estructural examina las relaciones entre la variable latente ( $\eta$ ) y las causas $\left(\mathrm{X}_{\mathrm{q}}\right)$ y el modelo de medición vincula los indicadores $\left(\mathrm{X}_{\mathrm{p}}\right)$ y la variable latente $(\eta)$. En el modelo MIMIC, la Soberanía Alimentaria es la variable latente $(\eta)$ y está determinada linealmente, sujeta a una perturbación $(\zeta)$ por un conjunto de causas exógenas observables como:

$$
\eta=\gamma_{1} x_{1}+\gamma_{2} x_{2}+\ldots \ldots+\gamma_{q} x_{q}+\zeta
$$

La variable latente $(\eta)$ determina linealmente, sujeto a las perturbaciones $\varepsilon_{1}, \varepsilon_{2}, \ldots \varepsilon_{p}$, el conjunto de indicadores observables como $y_{1}, y_{2}, \ldots y_{p}$ :

$$
\begin{gathered}
y_{1}=\lambda_{1} \eta+\varepsilon_{1} \\
\left\{y_{2}=\lambda_{2} \eta+\varepsilon_{2}\right. \\
y_{p}=\lambda_{p} \eta+\varepsilon_{p}
\end{gathered}
$$

De forma resumida en la figura 5 , se explica las fases que comprenderán el modelo MIMIC para la Soberanía Alimentaria en Ecuador.

Figura 5. Diagrama de flujo de las fases del Modelo MIMIC.

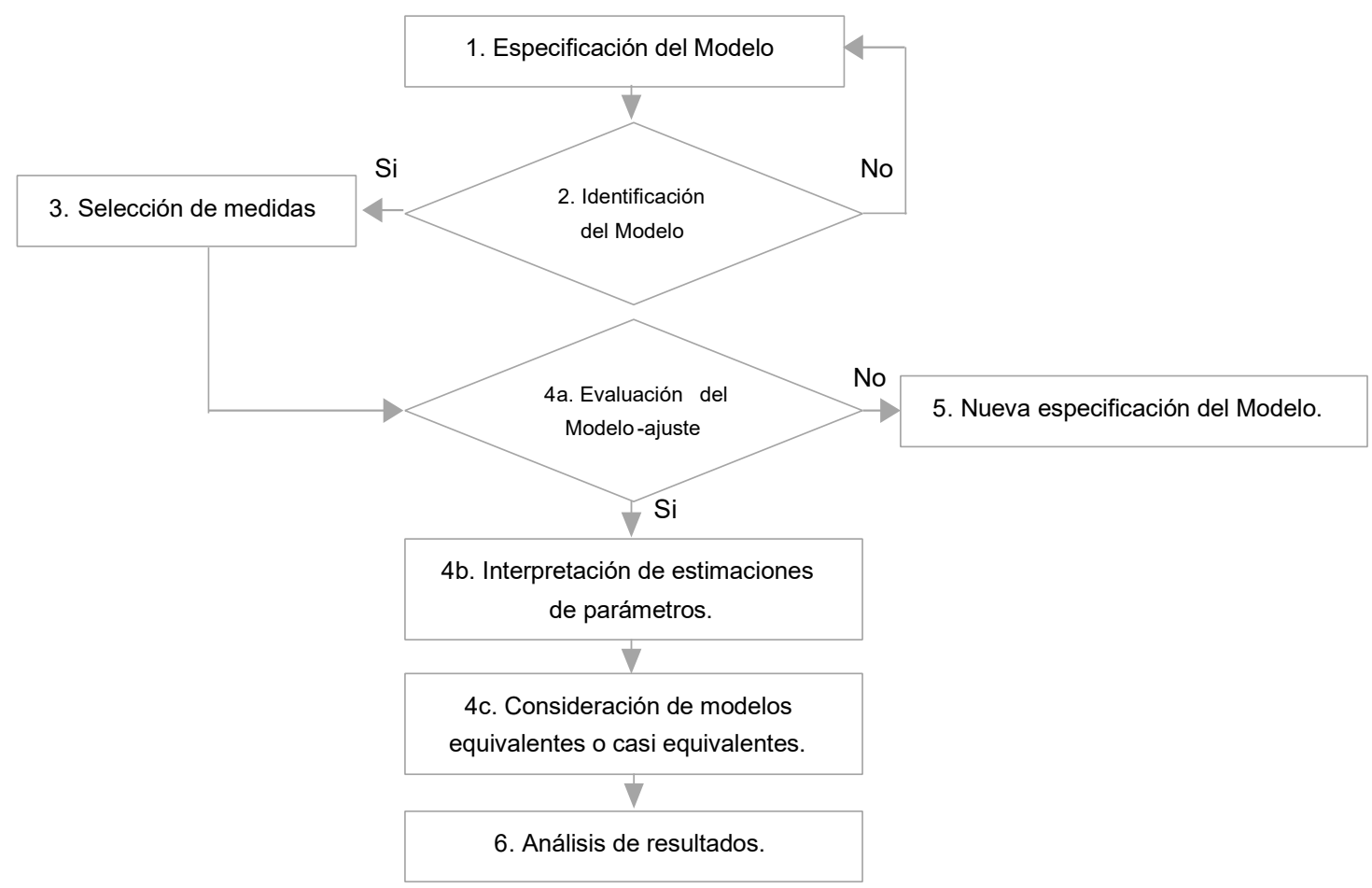

ISSN: 1390-9320, Edición Especial, diciembre 2019 
Fuente: Kline (2011)

Para la realización de la investigación se basa en seleccionar los indicadores que sean útiles para el territorio ecuatoriano, teniendo coherencia con la fundamentación teórica de la Soberanía Alimentaria, que tenga periodicidad vigente y de la base de datos anteriormente mencionada, también está tomada en investigaciones previas de indicadores donde se han realizado análisis de "350 grupos de indicadores, con una clasificación y subclasificación tras el análisis del concepto de Soberanía Alimentaria" (Ortega y Rivera, 2010), cuyo resultado fueron 128 indicadores en sus respectivas categorías y subcategorías.

\section{CONCLUSIONES Y RECOMENDACIONES}

Con base en a la revisión bibliográfica se puede concluir la necesidad de desarrollar la línea de investigación de un modelo de medición, para tomar medidas de políticas respecto al Derecho a la alimentación. El modelo de medición a proponer se puede situar en la teoría de la Soberanía alimentaria adaptándose a la realidad ecuatoriana, ya que tiene muchos lineamientos de entidades internacionales y ajuste de la teoría conceptual global.

El enfoque de Soberanía Alimentaria es una opción relevante para enfrentar los problemas de alimentación y contaminación, ya que dentro de esta propuesta está incluida la seguridad alimentaria. En el Ecuador la Soberanía Alimentaria absorbe, considera y evoluciona los problemas de la crisis alimentaria que ocurre desde el contexto global al local.

Asimismo, se orienta que el mejor modelo estadístico que se adapta a la línea de investigación es el enfoque Múltiples Indicadores y Múltiples Causas (MIMIC), por ser la variante más completa de los Modelos de Ecuaciones Estructurales (SEM) y que puede analizar la convergencia entre dos variables dependientes y múltiples variables independientes. De esta forma se analizan muchas variables indicadoras y las causantes del fenómeno de estudio.

También se puede concluir que la base de datos idónea para tomar información secundaria es la publicada en el Banco Mundial, ya que los organismos encargados del monitoreo de la contaminación y todo lo referente a temas de alimentación sustentan sus estadísticas en dicha base consolidada.

Como recomendación para futuras investigaciones se propone continuar con la línea de investigación y cumplir las fases del modelo MIMIC para poder dar resultados de convergencia 
sobre la Soberanía Alimentaria en Ecuador de forma piloto, para monitorear el fenómeno de investigación.

\section{REFERENCIAS BIBLIOGRÁFICAS}

Akchurin, M. (2015). Constructing the rights of nature: Constitutional reform, mobilization, and environmental protection in Ecuador. Law \& Social Inquiry, 40(4), 937-968.

Altieri, M. A. (2011). The agroecological revolution in Latin America: rescuing nature, ensuring food sovereignty and empowering peasants. Journal of Peasant Studies, 38(3), 587-612.

Anderson, M. D., \& Bellows, A. C. (2012). Introduction to symposium on food sovereignty: expanding the analysis and application. Agriculture and Human Values, 29(2), 177184.

Bernstein, H. (2015). Soberania alimentar: uma perspectiva cética. Sociologias, 276-336. Binimelis, R., Rivera-Ferre, M., Tendero, G., Badal, M., Heras, M., Gamboa, G., \& Ortega, M. (2014). Adapting established instruments to build useful food sovereignty indicators. Development Studies Research. An Open Access Journal, 1(1), 324-339.

Botella Rodríguez, E. (2018). Políticas agrarias, Seguridad Alimentaria y Nutricional y Soberanía Alimentaria: luces y sombras del caso cubano (1990-2015). Mundo Agrario, 19.

Butti Al Shamsi, K., Compagnoni, A., Timpanaro, G., Cosentino, S., \& Guarnaccia, P. A. (2018). Sustainable Organic Production Model for "Food Sovereignty" in the United Arab Emirates and Sicily-Italy. Sustainability, 10(3), 620.

Calix de Dios, H., Alvarado Dzul, S., Godek, W., Kissmann, S., Pierre, J. L., \& Gliessman, S. (2014). The challenges of measuring food security and sovereignty in the Yucatán Peninsula. Development in Practice, 24(2), 199-215.

Constitución del Ecuador, Registro Oficial 449 (Asamblea nacional del Ecuador 10 20, 2008). COPISA. (2019, 05 20). Conferencia Plurinacional e Intercultural de Soberanía Alimentaria. 
Retrieved from https://www.soberaniaalimentaria.gob.ec:

https://www.soberaniaalimentaria.gob.ec/prueba/servicios/mision-y-vision-3/

FAO. (2013). Ley Marco derecho a la alimentación, seguridad y soberanía alimentaria.

Panamá. Retrieved 01 06, 2019, from http://www.fao.org/3/a-au351s.pdf

FENOCIN. (2019, 05 20). Confederación Nacional de Organizaciones Campesinas

Indígenas y Negras. Retrieved from http://www.fenocin.org:

http://www.fenocin.org/sample-page/

Godek, W. (2014). The institutionalization of food sovereignty: the case of Nicaragua's law of food and nutritional sovereignty and security. 536. (Doctoral dissertation, Rutgers University-Graduate School-Newark).

Godfray, H. C., Beddington, J. R., Crute, I. R., Haddad, L., Lawrence, D., Muir, J. F., \& Toulmin, C. (2010). Food security: the challenge of feeding 9 billion people. science, 327(5967), 812-818.

Kline, R. (2011). Principles and Practice of Structural Equation Modeling. (3era ed.). New York, NY, USA: The Guilford Press.

Loke, M. K., \& Leung, P. (2013). Competing food concepts-Implications for Hawai'i, USA. Food and Energy Security, 2(3), 174-184.

López, L. A., y Franco, Á. (2015). Revisión de enfoques de políticas alimentarias: entre la seguridad y la soberanía alimentaria (2000-2013). Cadernos de Saúde Pública, $31,1355-1369$.

LORSA, Ley Orgánica del Régimen de la Soberanía Alimentaria (Registo Oficial Suplemento 58305 05, 2009).

MAGAP. (2019, 05 20). Ministerio de Agricultura, Ganadería, Acuacultura y Pesca. Retrieved from https://www.agricultura.gob.ec: https://www.agricultura.gob.ec/elministerio/

ONU. (2019). Organización de las Naciones Unidas. Retrieved 01 06, 2019, from https://www.un.org: https://www.un.org/sustainabledevelopment/es/objetivosdedesarrollo-sostenible/ 
Ortega, M., \& Rivera, M. G. (2010). Indicadores internacionales de Soberanía Alimentaria: nuevas herramientas para una nueva agricultura. Revibec: revista de la Red Iberoamericana de Economia Ecológica, 14, 53-77.

Parlamento Latinoamericano, C. (2012). Proyecto de Ley Marco "El Derecho a la Alimentación y Soberanía Alimentaria". Buenos Aires. Retrieved 01 06, 2019, from http://parlatino.org/pdf/leyes_marcos/leyes/proyecto-derecho-alimentacionsoberaniapma-30-nov-2012.pdf

Rosset, P. M. (2016). La reforma agraria, la tierra y el territorio: evolución del pensamiento de La Vía Campesina. Mundo agrario, 17.

SENPLADES. (2017, 09 22). Plan Nacional de Desarrollo 2017-2021. San Francisco de Quito. Retrieved 01 06, 2019, from http://www.planificacion.gob.ec/wpcontent/uploads/downloads/2017/10/PNBV-26OCT-FINAL_OK.compressed1.pdf

Simon Reardon, J. A., \& Pérez, R. A. (2010). Agroecology and the development of indicators of food sovereignty in Cuban food systems. 34 (8).

Trebicka, B. (2014). MIMIC model: A tool to estimate the shadow economy. Academic Journal of Interdisciplinary Studies, 3(6), 295.

Villegas, L. F. (1998). Estado, cuestión agraria y movilización india en Ecuador. Los desafíos de la democracia. Nueva Sociedad, 56-72. 